\title{
光滑壁面明渠均匀案流水力特性*
}

\author{
董曾南 丁 元 \\ (清华大学水利系,北京)
}

\begin{abstract}
摘 要
水流进入光滑壁面明渠后,沿水流方向历经层流边界层，转挨区，紊流边界层，充 分发展紊流边界层及明渠均勺紊流等流动区域。明渠均勺紊流中流动的时均及紊动 特性均不再沿程变化. 光滑壁面明渠均匀紊流中，沿水深方向流动具有明显的分区 结构，本文给出了分区界限及各分区中时均流速分布公式及脉动流速的特征。本文 提出当明渠的宽深比 $W / H$ 大于 10 时方可将中心区流动视为二维流动, 并给出了断 面平均流速及阻力系数的计算公式。
\end{abstract}

\section{关键词: 明渠水流,水力特性,美流}

\section{一、引 言}

河流、渠道及水工建筑物中的流动大多是具有自由水面的充分发展的紊流边界层流动,即 明渠紊流。明渠紊流中当其流动要素不再随流程而变化时形成明渠约匀紊流。自 1775 年以 来,工程实践中明渠均匀紊流的计算一直采用 Chézy 总结的经验公式 $\nu=c \sqrt{ } R \vec{J} .1938$ 年 Keulegan ${ }^{[1]}$. 得到了光滑与粗䊁壁面明渠均匀紊流的时均流速分布公式. 但是随着生产的发展 和科学的进步，人们需要解决更深人一步的问题，即流动要素在空间上和时间上的逐点分布。 如泥沙运动、河流中污染物质的扩散与弥散、水工建筑物中的渗气、空虻、脉动压力等高速水流 问题都不仅要了解水流运动的时均流速特性与分布而且要深人了解其紊动特性. 因而对明渠 紊流的研究提出了新的要求.

近 20 年来很多学者使用热膜流速仪及氢泡显示技术对明渠紊流做了大量的试验研究工 作, 但是由于使用热膜流速仪在水中进行紊流量测还存在一定的困难,量测结果达不到象热丝 风速计在气流测量中所达到的精度. 80 年代以来有些学者开始使用激光测速仪 (LDV) 进行 明渠紊流的研究 ${ }^{[2-4]}$. 但由于流动本身的复杂性, 目前在很多问题上尚未得出公认的结论.

本文使用自制激光测速仪及实时数据采集处理系统对明渠均匀紊流水力特性进行了系统 的试验研究. 由于仪器的空间分辨率高, 抗干扰能力强, 因而可以精细地测量紧贴固体壁面处 的紊流流速场, 得出一些有价值的结论, 如明渠中形成均匀紊流的过程; 明渠均匀紊流的流速 分区结构, 分区的界限及各分区流动特性; 明渠均匀紊流的流速及阻力公式; 明渠均匀紊流的

本文1988尔11月1日收到, 1989 年4月4日收到修改稿.

*国家自然甜学基金资助项目. 
紊动特性,壁面切应力以及明渠流动二维性的判据。

\section{二、试验设备仪器与方法}

本试验研究是在特制玻璃水槽中进行，水槽长 $7 \mathrm{~m}$, 宽 $25 \mathrm{~cm}$ ，高 $30 \mathrm{~cm}$. 首部设稳水箱， 尾部设有可调尾门. 试验用明渠渠底为精制铝板，表面进行烤澡处理以减小激光测量体接近 固体壁面时的反射及干犹，水槽两侧为玻璃，渠底铝板粗䊁度经测量为 $\kappa_{s}<10 \mu \mathrm{m}$. 本试验 中最大的粗粘雷诺数 $\left(\frac{\kappa_{s} u_{*}}{v}\right)_{\max }=0.2 \ll 5$, 因而说明渠道为水力光滑壁面. 为减小水流进 人明渠后的水面跌落并使水面稳定, 铝板较玻璃水槽槽底高 $5 \mathrm{~cm}$, 铝板头部做成流线形, 铝板 上下均有水流通过，上面形成明渠流动。渠底坡度为 $1.3 \%$ ，本文采用右手坐标系，顺流方向 沿铝板中心线为 $x$ 轴, 垂直槽底向上为 $y$ 轴, 展向为 $z$ 轴. 坐标原点设于平板前缘中心处.

流速量测采用实验室自制的偏振差动式激光测速系统，系统中设置了光束扩展器，光学 渍波和电子差动电路,因而具有动态响应快,空间分辨率高,抗干犹能力强,在近壁面处可消除 强背景噪音的特点. 数据采集与处理采用 JSP - 1 计数型信号处理系统, 此系统在流速较低, 水中粒子浓度较小的情况下仍然具有良好的工作性能. 流速信号通过数据处理系统直接给出 所有的紊动统计量. 本试验中, 激光测速系统与高精度的走行机构相配合, 可量测壁面附近 $30 \mu \mathrm{m}$ 处的时均及脉动流速.

试验中水面量测使用精度为 $0.1 \mathrm{~mm}$ 的测针, 流量量测使用三角堰.

本试验对 7 种水深情况进行了系统的测量, 水流情况见表 1 .

表 1 试验水流条件

\begin{tabular}{|c|c|c|c|c|c|c|}
\hline 序号 & $\begin{array}{c}\text { 水深 } \\
H(\mathrm{~mm})\end{array}$ & $\begin{array}{l}\text { 宽深比 } \\
W / H\end{array}$ & $\begin{array}{c}\text { 单宽流量 } \\
q \\
\left(\mathrm{~mm}^{3} / \mathrm{s}, \mathrm{mm}\right)\end{array}$ & $\begin{array}{c}\text { 断面平均流速 } \\
v \\
(\mathrm{~mm} / \mathrm{s})\end{array}$ & $\begin{array}{l}\text { 霓诺数 } \\
\operatorname{Re} \theta \\
\left(\frac{\nu_{R}}{\nu}\right)\end{array}$ & $\begin{array}{c}\text { 佛汝德数 } \\
F_{r} \\
\left(\frac{v}{g H}\right)\end{array}$ \\
\hline 1 & 15 & 16.67 & $3.5 \times 10^{3}$ & 236 & $2.8 \times 10^{3}$ & 0.62 \\
\hline 2 & 20 & 12.50 & $6.1 \times 10^{3}$ & 303 & $4.6 \times 10^{3}$ & 0.68 \\
\hline 3 & 25 & 10.00 & $8.2 \times 10^{3}$ & 328 & $6.0 \times 10^{3}$ & 0.66 \\
\hline 4 & 30 & 8.33 & $1.07 \times 10^{4}$ & 358 & $7.6 \times 10^{3}$ & 0.66 \\
\hline 5 & 36 & 6.94 & $1.45 \times 10^{0}$ & 404 & $9.9 \times 10^{3}$ & 0.68 \\
\hline 6 & 40 & 6.25 & $1.69 \times 10^{4}$ & 422 & $1.1 \times 10^{4}$ & 0.67 \\
\hline 7 & 46 & 5.43 & $2.09 \times 10^{4}$ & 454 & $1.3 \times 10^{\circ}$ & 0.68 \\
\hline
\end{tabular}

$R$ 一水力半径.

ע-D水流运动粘滞系数.

\section{三、试验结果.与分析}

\section{1. 明渠均匀亲流的形成}

根据沿程各过水断面的流速分布特点,水流进入明渠后其发展过程沿流程河分为: 层流 边界层,转㨭区、紊流边界层, 充分发展紊流边界层(明渠紊流), 明渠均匀紊流 5 个阶段。

水流进人铅板与两侧玻璃所形成的渠道后,首先为层流边界层流动。此时时均流速在断 面上的分布可分为两部分：边界层内的流速分布基本符合 Blasius 平汳层流边界层理论解， 
边界层外流速均与分布为势流区. 随着流程的增加, 边界层逐渐增厚, 边界层内的流速分布逐 渐偏离 Blasius 解, 流动进入转挓区. 流程继续增加, 流动由转㧖区进入紊流边界层流动. 图:

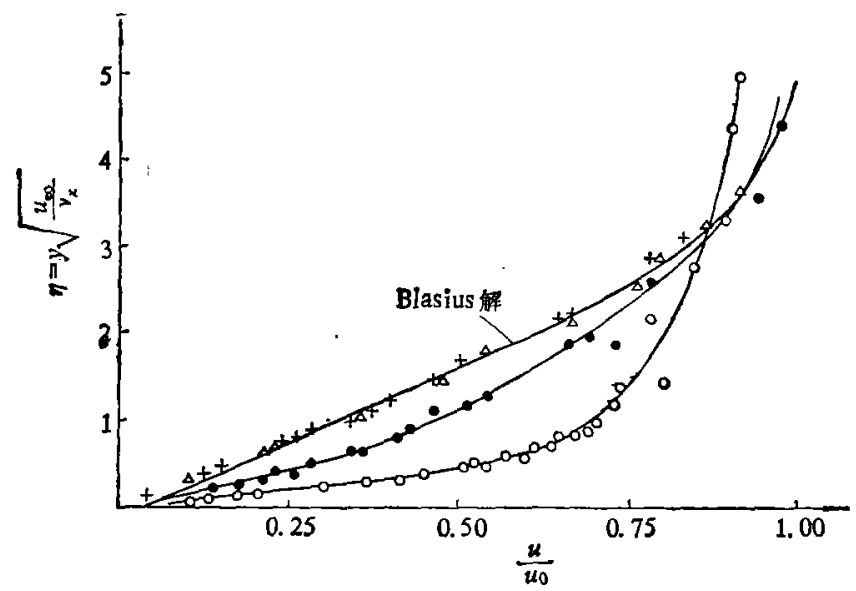

图 1 流速分布与 Blasius 解

$(H=20 \mathrm{~mm} ; \Delta$ 表示 $x=0.02 \mathrm{~m}$ (层流边界层)，十表示 $x=0.2 \mathrm{~m}$ (层流边

界层), 表示 $x=0.25 \mathrm{~m}$ (转捩区), 0 表示 $x=0.8 \mathrm{~m}$ (紊流边界层))

1 为层流边界层、转㨭区、 紊流边界层 3 个区域的典 型流速分布与 Blasius 解 的对照.

判断紊流边界层的起 始位置须综合研究以下因 素:

（1）流速分布的形状 参数 $H_{12}=\frac{\delta_{1}}{\delta_{2}}$ 在层流边界 层中约为 2.6 , 进入转㙆区 $H_{12}$ 明显下降, 至紊流边界 层由于流速分布图形与层 流边界层明显不同, $H_{\mathrm{n}}$ 下

降至 1.4 左右, 如图 2.

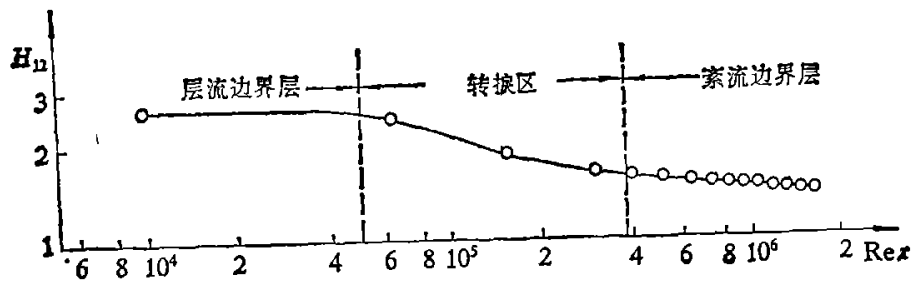

图 2 形状参数 $H_{12}$ 与雷诺数 $\operatorname{Re} x$ 的关系曲线 (水深 $36 \mathrm{~mm}$ )

（2）边界层内流速分布可用对数律表示为

$$
u^{+}=\frac{1}{K} \ln y^{+}+B
$$

或用指数律表示为

$$
u^{+}=C\left(y^{+}\right)^{1 / n}
$$

式中 $u^{+}=\frac{u}{u_{*}}, y^{+}=\frac{y u_{*}}{v}, u$ 为一点流速, $u_{*}=\sqrt{\frac{\tau_{0}}{\rho}}$ 为壁面剪切流速, $\tau_{0}$ 为壁面切应力, $\rho$ 为水的密度. $K$ 为 Kármán 常数, 是表示壁面附近紊动特征的一个普适常数. $B$ 为由固体壁面 表面特征所确定的一个常数. $C, n$ 为表示流速分布的常数. 图 3、图 4 表示这些常数和指数 沿流程的变化. 可以看出在转掞区 $K, B$ 均呈上升趋势, 进入紊流边界层后 $K, B$ 趋于稳定。 $C, n$ 在转挓区比较分散, 至紊流边界层后则逐渐上升, 进入明渠均匀紊流区域则形成稳定值, 不再变化.

紊流边界层中由粘性底层至紊流层之间的过渡层中流速分布也可用指数形式表示如下: 


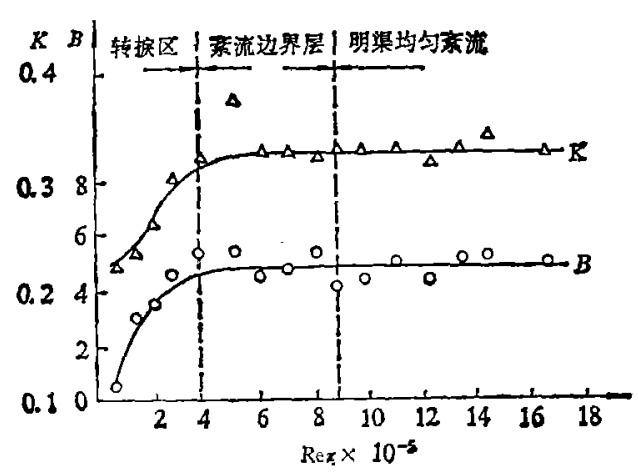

图 3

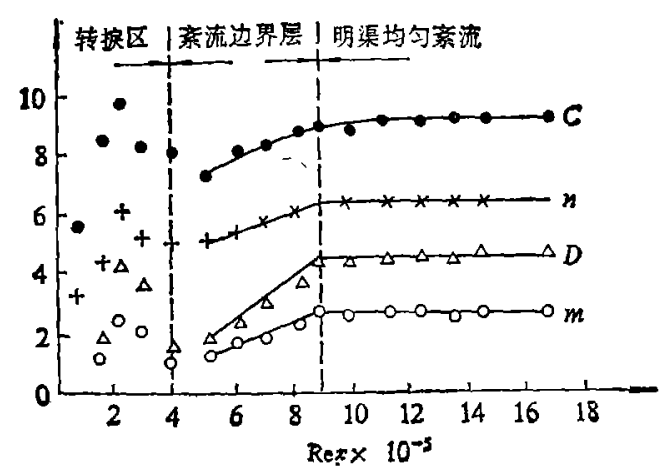

图 4

$$
u^{+}=D\left(y^{+}\right)^{1 / m} .
$$

常数 $D$ 与 $m$ 的变化规律与 $C, n$ 相同, 如图 4.

（3）边界层厚度随流程的发展规律在各区内明显不同. 在层流边界层内边界层厚度随雷 诺数 $\operatorname{Re} x$ 的增长符合 Blasius 理论解， $\delta=5 \frac{x}{\sqrt{\operatorname{Re} x}}$, 在紊流边界层中边界层厚度的发展 规律与无界流场中光滑平板紊流边界夽厚度公式:

$$
\begin{gathered}
\delta^{5 / 4}-\delta t^{5 / 4}=0.29\left(\operatorname{Re} x^{-1 / 4} x^{5 / 4}\right. \\
\left.-\operatorname{Re} x_{t}^{-1 / 4} x_{t}^{5 / 4}\right)
\end{gathered}
$$

相比, 厚度发展较缓慢, 如图 5 所 示.（4）式中下标 $t$ 表示转挨区进 人紊流区处的有关数值. 图 5 中表 示水深 $H$ 为 $36 \mathrm{~mm}$ 及 $46 \mathrm{~mm}$ 两种情 况,具有相同的规律性.

当边界层厚度增加到与水深相 等后, 开始进人充分发展案流边界 层区域,此时称为明渠紊流, 其水力 要素仍在沿程变化. 明渠紊流需视 明渠的底坡、壁面状况、流量、水 深等因素而逐渐发展为明渠均匀紊 流。进人明渠均匀紊流后，水流的 各种水力要素才不再沿程变化, 呈

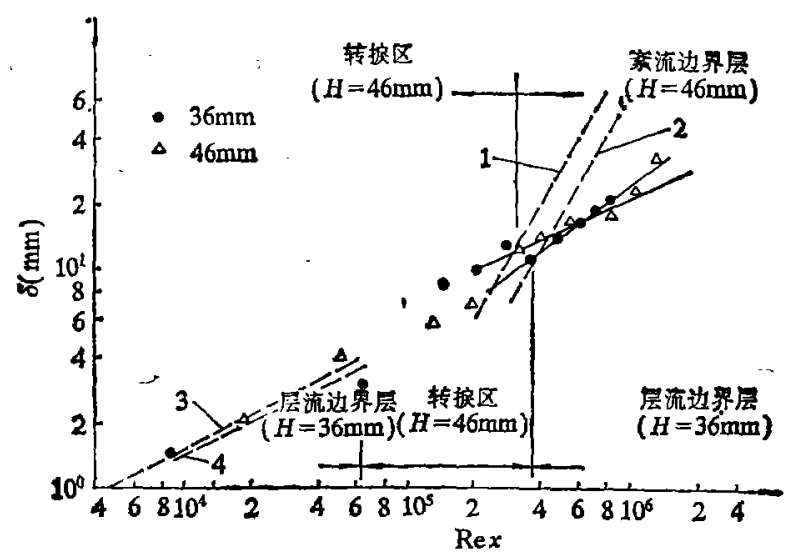

图 $5 \delta \sim \operatorname{Re} x$ 关系图

$$
\begin{gathered}
(1-\text { 公式(4) }(H=46 \mathrm{~mm}), 2 \text { - } \text { 公式(4) }(H=36 \mathrm{~mm}), \\
3-\delta=5 x / \sqrt{\operatorname{Rex}}(H=36 \mathrm{~mm}), 4-\delta=5 x / \sqrt{\operatorname{Rex}} \\
(H=46 \mathrm{~mm}))
\end{gathered}
$$

现均匀流的性质. 由图 4 可看出这一点. 在水流进入明渠均匀紊流后断面平均流速点的位置 $\bar{y}=\alpha H$, 断面平均紊流度也都不再沿程变化. 明湈均匀紊流中 $\alpha=0.36($ 图 6,7).

\section{2. 明渠均匀亲流的分区结构}

明渠均匀紊流中的时均流速分布由于其在工程实践和理论中的重要性，一直为人们所关 注. 本文通过大量量测得出在明渠均匀紊流中, 沿水深可分为粘性底层、过渡层及紊流层 3 个 区域。 


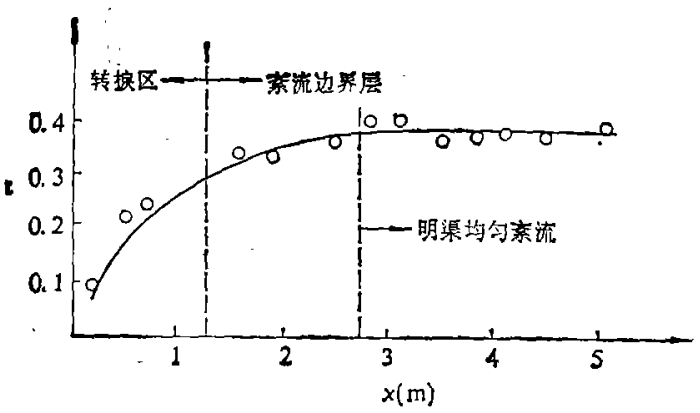

4

图 $6 \alpha$ 沿程变化曲线(水深 $36 \mathrm{~mm}$ )

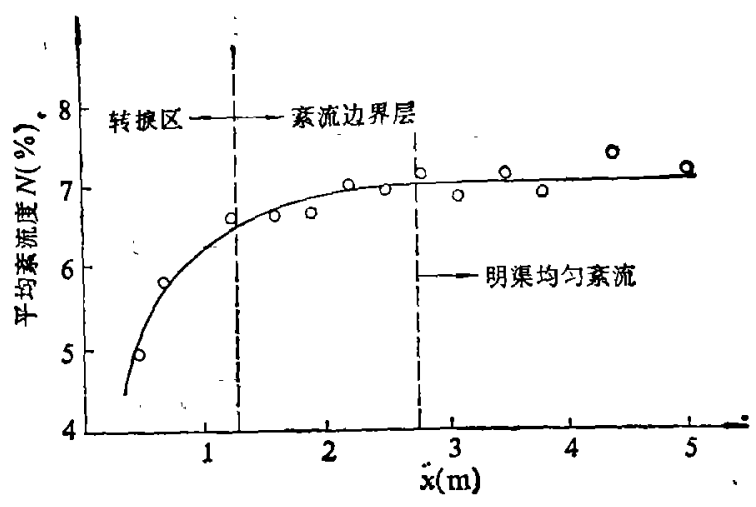

图 7 平均紊流度沿程变化曲线 (水深 $36 \mathrm{~mm}$ )

（1）粘性底层 研究光滑壁面明渠均匀紊流的流速分布首先要解决如何精确确定壁面 零点 $y_{0}$ 和壁面剪切流速 $u_{*}$ 的问题．本文使用文献[3]的方法得出在明渠水流粘性底层中流 速分布公式为

$$
\frac{u}{u_{*}}=\frac{y u_{*}}{\nu} \text {, 或写为 } u^{+}=y^{+} \text {. }
$$

通过对大量断面量测资料的分析得出粘性底层的厚度为

$$
\delta_{*}=(8-10) \frac{\nu}{u_{*}}
$$

（2）亲流层 在明渠中沿水深大部分为䒺流层, 紊流层的流速分布自 Prandt1 以来一 致认为符合对数律; 即

$$
\frac{u}{u_{*}}=\frac{1}{K} \ln \frac{y}{\nu} \underline{u}_{*}+B
$$

在半对数流速分布图中 (图 8)， $K$ 表示对数分布曲线的斜率而 $B$ 则表示该线的截距. 对于光 滑壁面管道，Nikuradse 得到 $K=0.4 ， B=5.5$. 不同研究者所得到的 $K$ 的数值多有出人， 大致在 $K=0.37-0.47$ 之间. 对明渠以往则多沿用 Nikuradse 的结果.

本文中对紊流区的界限采用试算方法,使回归计算得到最大的相关系数, 从而得到的紊流 层界限为

$$
y_{t}^{+} \geqslant(22-24) \text {. }
$$

对于紊流层的上限, Nezu 和 $\operatorname{Rodi}^{[2]}$ 认为在 $y / h=0.2$ 处, $y / h>0.2$ 则为尾流律区 域. 但从他们的文章中所发表的流速图形看有些水流条件下尾流律并不明显. 在整个水深上 (除粘性底层与过渡层)用对数律表示流速分布并不会引起过大误差. 本文中进行的大量量测 同样得到这样的结论,对于大多数断面而言对数律可以延伸到接近自由水面处（图 8). 只在 个别断面上有尾流律区域的存在.

表 2 列出了各种不同水深条件下的量测成果并给出了综合的平均值. 表中 $\delta_{B \alpha}^{+}$为无量纲 过渡层上边界位置， $\delta_{3}^{+}$为无量纲过渡层厚度。由表 2 得出紊流层的流速分布可写为

$$
u^{+}=6.12 \log y^{+}+5.49 \text {, }
$$


即 $K=0.376, B=5.49$. 与文献[3]在明流平板边界层中所得 $K=0.39, B=5.48$ 基本一

致. 用指数律表示则

$$
u^{+}=8.77\left(y^{+}\right)^{1 / 6.65},
$$

与圆管紊流 中 ${ }^{[5]} c=8.74, n=7$ 略有差别.

（3）过渡层 在粘性底层与 紊流层之间存在一过渡层, 其上下 范围为

$$
(8-10) \leqslant \frac{y u_{*}}{\nu} \leqslant(22-24),
$$

与圆管紊流相比，明渠紊流的粘性 底层较厚, 圆管中过渡层在

$$
5 \leqslant \frac{y u_{*}}{v} \leqslant(30-70),
$$

明渠中过渡层较薄。根据本文试验 成果过渡层中流速分布可表示为

$$
u^{+}=12.8 \log y^{+}-3.42
$$

或 $u^{+}=3.56\left(y^{+}\right)^{1 / 2.31}$.

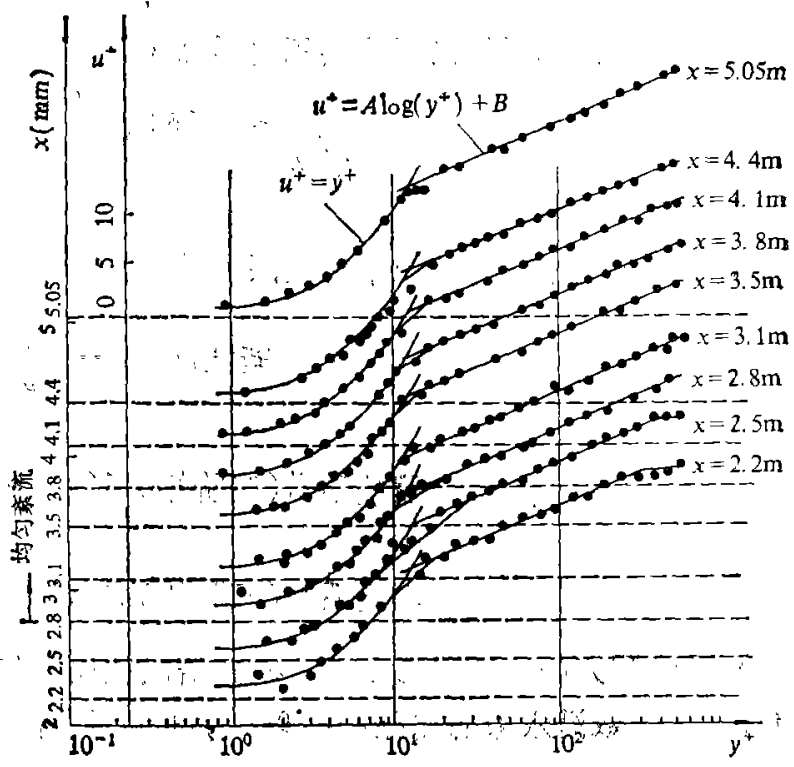

图 8 流速分布图

\begin{tabular}{|c|c|c|c|c|c|c|c|c|}
\hline 项目 & 15 & 20 & 25 & 30 & 36 & $\begin{array}{r}40 \\
-\end{array}$ & 46 & 平均值 \\
\hline$K$ & 0.398 & 0.415 & 0.374 & 0.387 & 0,336 & 0.378 & 0.341 & 0.376 \\
\hline$B$ & 5.98 & 5.60 & 5.16 & 5.61 & 5.25 & 5.15 & 5.65 & 5.49 \\
\hline$c$ & 8.75 & 8.55 & 8.70 & 8.79 & 9.06 & 8.85 & 8.86 & 8.77 \\
\hline$n$ & 6.47 & 6.71 & 6.58 & 6.72 & $6: 43$ & 6.80 & 6.85 & 6.65 \\
\hline$D$ & 3.51 & 4.25 & 2.49 & 3.25 & 4.58 & 3.09 & 3.77 & 3.55 \\
\hline$m$ & 2.34 & 2.64 & 1.75 & 2.1 .2 & 2.63 & 2.15 & 2.55 & 2.31 \\
\hline$\alpha$ & 0.35 & 0.36 & 0.36 & 0.37 & 0.37 & 0.35 & 0.37 & 0.36 \\
\hline$\delta_{0}^{+}$ & 7.73 & 7.09 & 7.38 & 7.54 & 10.8 & 8.42 & 8.93 & 8.27 \\
\hline$\delta_{B_{*}^{*}}^{+}$ & 23.9 & 21.5 & 18.7 & 21.9 & 18.6 & 20.8 & 26.2 & 21.8 \\
\hline$\delta_{B}^{+}$ & 16.2 & 14.4 & 11.3 & 14.4 & 7.8 & 12.4 & 17.3 & 13.4 \\
\hline$H_{12}$ & 1.47 & 1.45 & 1.43 & 1.41 & 1.40 & 1.39 & 1.39 & 1.42 \\
\hline$\delta_{B}^{+} / \delta_{b}^{+}$ & 2.1 & 2.0 & 1.5 & 1.9 & 0.7 & 1.5 & 1.9 & 1.7 \\
\hline
\end{tabular}

Nikuradse 在圆管中得到过渡层流速分布为 $u^{+}=11.5 \log y-3.05^{[6]}$, 可见明渠中常数均稍 大.

表 2 各种水流条件下成果总表

由以上讨论可知明渠均匀紊流中流速分布确有分区结构，各分区的界限和分区中流速分 布公式与圆管紊流近似，但数值有差异，与明流边界层的情况 ${ }^{[3]}$ 相比，则更为接近. 或者说水 流情况在明流紊流边界层中与明渠均匀案流中基本一致。

忽略极薄的粘性底层与过渡层,将(8)式沿全断面积分可计算明渠均匀紊流的断面平均流 
速

$$
\nu=u_{*}\left[6.12 \log \frac{H u_{*}}{\nu}+2.86\right],
$$

从而可得光滑壁面明渠的沿程水头损失系数 $\lambda$

$$
\frac{1}{\sqrt{\lambda}}=2.16 \log \frac{H u_{*}}{\nu}+1 .
$$

\section{3. 明渠均匀美流的紊动特性}

本文研究了明渠均匀紊流中沿水深和沿流程的紊动特性,定义如下:

$$
\text { 紊流度 } N=\frac{\sqrt{\vec{u}^{2}}}{v} \text {, }
$$

$$
\begin{aligned}
& \text { 偏差系数(三阶矩) } S(u)=\frac{\sqrt[3]{\bar{u}^{\prime 3}}}{v}, \\
& \text { 蜂凸系数(四阶矩) } F(u)=\frac{\sqrt[4]{4 \overline{u^{4}}}}{v} \text {. }
\end{aligned}
$$

（1）沿水深方向的亲动特性本文给出明渠均匀紊流中 4 个水深情况下的紊流度，偏 差系数,峰凸系数沿水深的变化如图 9,10,11 所示. 由图可以看出,在不同分区中紊流特性明

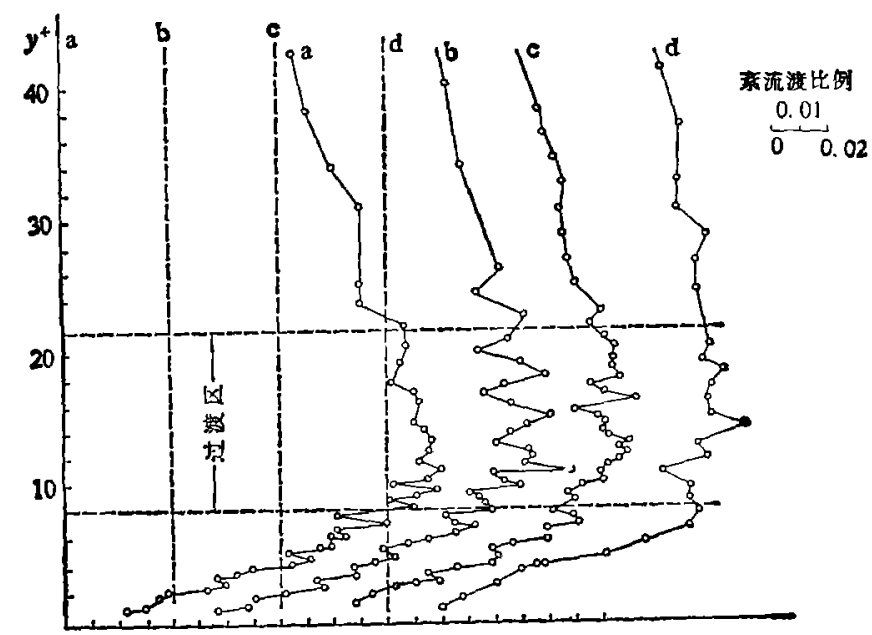

图 9 紊流度分布图

$$
\begin{gathered}
\text { (a: } H=15 \mathrm{~mm}, x=4.85 \mathrm{~m} ; \mathrm{b}: H=20 \mathrm{~mm}, x=5.00 \mathrm{~m} ; c: H=30 \mathrm{~mm}, x=4.85 \mathrm{~m} ; \\
\mathrm{d}: H=40 \mathrm{~mm}, x=4.80 \mathrm{~m} ; \\
\text { 编号虚线为对应曲线的基线） }
\end{gathered}
$$

显不同. 在壁面附近紊流度及峰凸系数沿高程急剧单调递增, 偏差系数为正值是为粘性底层. 在流场上部紊流层内紊流度和峰凸系数缓慢减小,偏差系数分布较为均匀目均为负值.二者 之间的过渡层内紊流度和峰凸系数均出现最大值并呈现复杂的大幅度随机变化，数值很不稳 定. 偏差系数在此区域内出现跳跃、变号、数值不稳定.

图 12 为粘性底层及紊流区中典型的流速概率分布曲线。可以清楚地看出粘性底层中概 


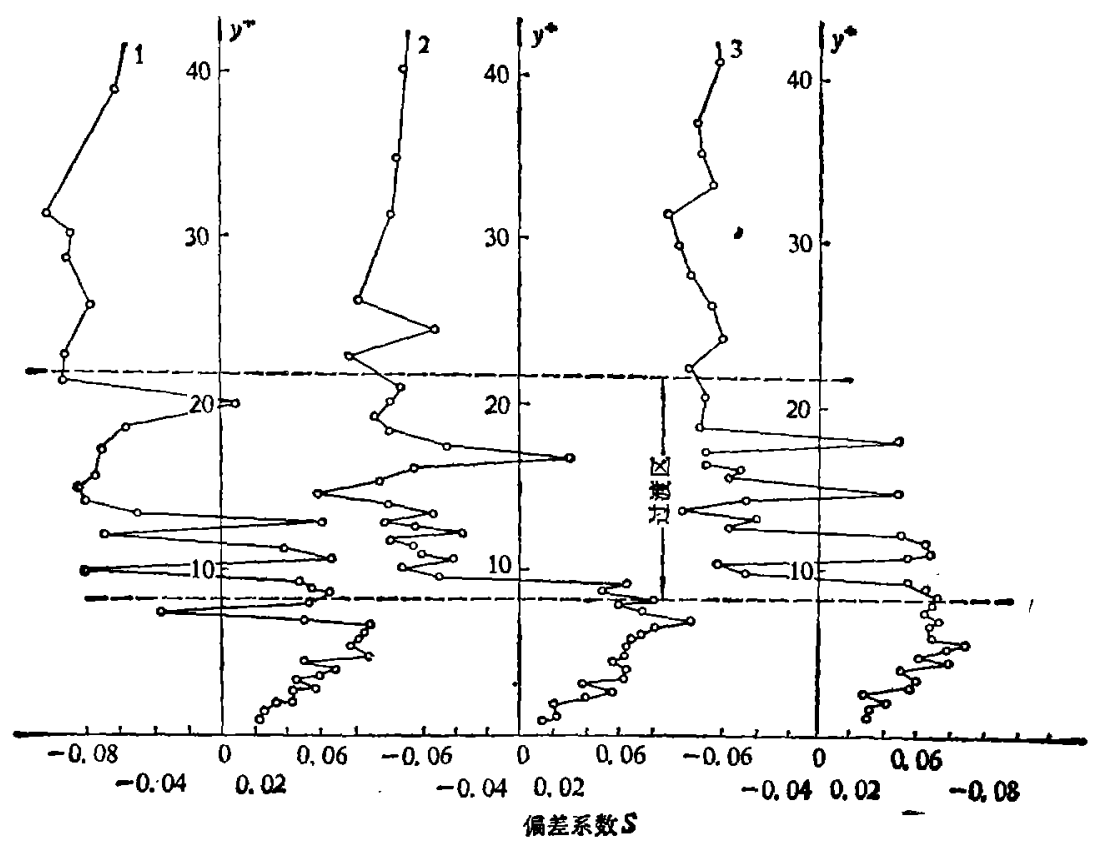

图 10 偏差系数 $S$ 分布

$$
\begin{gathered}
(1-\mathrm{a}: H=15 \mathrm{~mm}, x=5.00 \mathrm{~m} ; 2-b: H=20 \mathrm{~mm}, x=5.00 \mathrm{~m} ; 3-c: H=30 \mathrm{~mm}, \\
x=5.00 \mathrm{~m})
\end{gathered}
$$

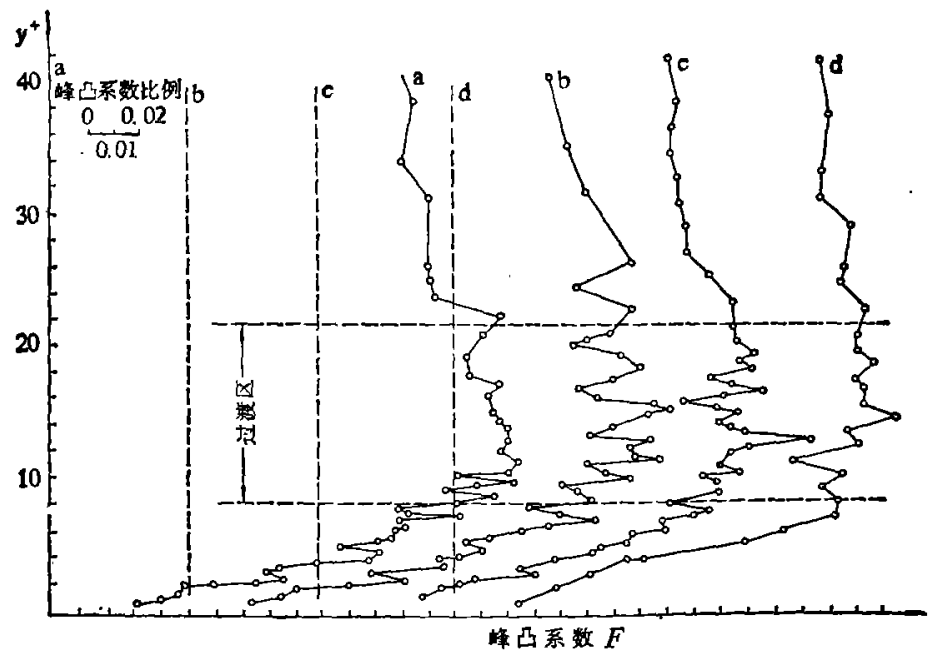

图 11 峰凸系数 $F$ 分布

(a: $H=15 \mathrm{~mm}, x=4.85 \mathrm{~m} ; \mathrm{b}$ : $H=20 \mathrm{~mm}, x=5.00 \mathrm{~m} ; c$ : $H=30 \mathrm{~mm}, x=4.85 \mathrm{~m} ; \mathrm{d}$ : $H=40 \mathrm{~mm}, x=4.80 \mathrm{~m}$; 编号虚线为对应曲线的基线）

率的峰值向左偏, 对应于正的偏差系数. 在紊流区内概率峰值向右偏,对应于负的偏差系数. 将偏差系数变号的区间进行统计平均, 得到其范围为

$$
9<y^{+}<21 \text {. }
$$

与(10)式中由时均流速确定的过渡区范围完全一致. 


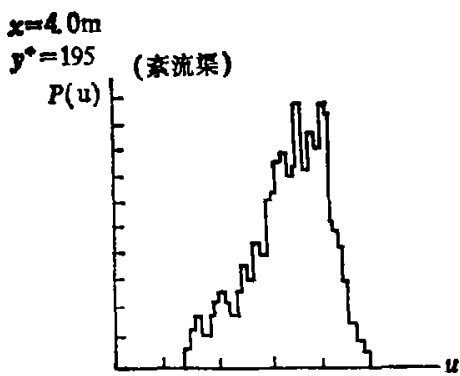

(a)

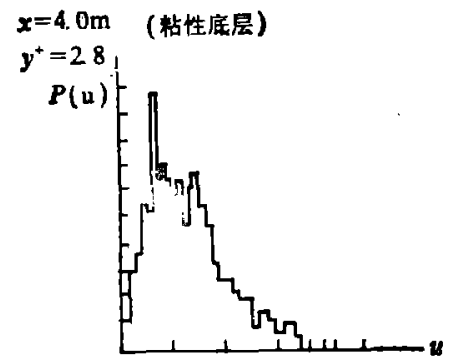

(b)

图 $12 \cdot$ 紊流区及粘性底层的概率分布曲线

$(H=20 \mathrm{~mm})$

紊流度与峰凸系数出现最大值的位置基本重合，对各断面进行平均，得到该点位置的无 量纲高度 $y^{+}=13.7$, 显然此点位于过渡区内. 断面最大紊流度,$N_{\max }=0.14$. 此结果与 Laufer ${ }^{[7]}$ 使用热线风速仪研究气流的充分发展紊流圆管流动所得 $y^{+}=14$ 时顺流方向流速 紊动度达到最大值的结论一致。

由以上分析司以看出在明渠均匀紊流中沿水深有分区结构，不同的流层中不仅时均流速 分布而且紊动特性也具有不同的特点。

（2）亲动特性沿流程的变化 图 7 表示了断面平均紊流度沿流程的变化规律. 图中表 明在由层流边界层到紊流边界层之间的转㧖区断面平均紊流度沿程上升较快。进入紊流边界 层区域上升趋势减缓。进入明渠均匀紊流区域后断面平均紊流度不再变化: 为 0.07 去: 右.

（3）改变水深时的亲动特性 在明渠均匀紊流区域，不同水深情况下紊流度，偏差系 数, 峰凸系数的断面平均值基本不变而保持常数, 其统计平均值分别为

\section{4. 壁面切应力}

$$
\begin{aligned}
& N=(7.0 \pm 0.5) \%, \\
& S=(-3.9 \pm 0.5) \%, \\
& F=(9.0 \pm 0.6) \% .
\end{aligned}
$$

壁面切应力形成水流阻力, 使水流运动产生水头损失. 由壁面切应力定义的剪切流 速 $\boldsymbol{u}_{*}=\sqrt{\frac{\tau_{0}}{\rho}}$ 对边界层内的流速分布以及紊流特性都有着重要的影响, 因此受到工程界及理论 研究者的重视.

由前面的分析已经可以看出在明渠均匀紊流流动中与管流存在某些相似，特别是在近壁 区, 时均流速的分布, 紊动特性以及分区结构均有相当的近似之处. 但在明渠中由于自由水面 的存在,渠道断面非圆形而形成水流的三维性, 沿渠道湿周壁面切应力的非均匀分布等因素, 又使明渠流动与管流有很大的区别, 特别是在自由水面附近和角隅区域. 有些学者认为当明 渠的宽深比 $W / H$ 大于某一数值,例如 $5-6^{[2,8]}$ 则在明渠中存在某一中心区域, 这个区域的水 流可以认为是二维流动. 对于宽深比很大的渠道, 底面切应力分布较均匀, 侧壁影响消失 ${ }^{[0]}$, 形成二维流动,这时的明渠水流与管流的区别只是自由水面的存在.

壁面切应力的确定通常有两种方法,一是直接测量的方法如使用 Preston 管, 热膜式或应 变式传感器等. 二是间接量测的方法, 即由明渠的水力坡度计算, 或由雷诺应力沿水深分布延 
呻至壁面处确定,或由紊流区时均流速的对数分布公式,选择最佳适配常数 $u_{*}$ 及壁面零点高 程 $y_{0}$ ，再由 $u_{*}$ 确定壁面切应力值，以及本文中用粘性底层流速分布计算切应力

$$
\tau_{0}=\left.\mu \frac{d u}{d y}\right|_{,=0}
$$

等。

直接测量方法由于仪器对壁面附近粘性底层中同部水流的干扰, 因而量测的结果不易准 确. 间接测量方法中，水面坡度的量测往往不易精确，由雷诺应力的线性分布延伸至壁面所 得的 $\tau_{0}$ 值往往大于实际壁面粘性切应力, 利用流速的对数分布律则必须承认 Kármán 常数 $K=0.4$ 及 $B$ 的数值, 而事实上 $K, B$ 的数值是有一个变动范围的.

本文采用激光量测粘性底层中流速分布，是一种无干扰的量测方法，因而所得数值比较可 靠.

以壁面切应力作为明渠流动是否可近似视作二维流动的判据则要求切应力在底面沿展向

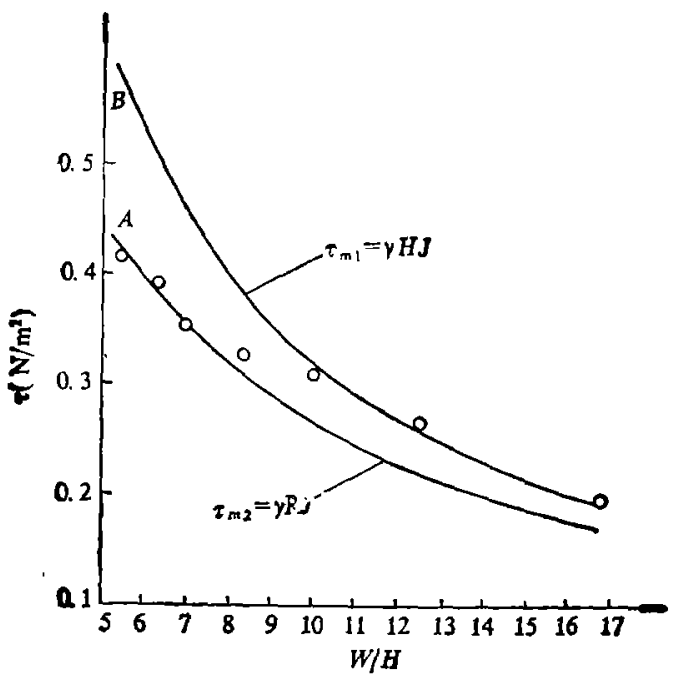

图 $13 \tau_{0}$ 对宽深比的分布

分布均匀, 侧壁影响可以忽略, 此时 $\tau_{0}$ 应与

$$
\tau_{m_{1}}=r H J
$$

相同,而对于明渠三维流动则壁面沿湿周的平均切应力应为

$$
\tau_{\mathrm{m}_{2}}=r R J \text {. }
$$

图13中给出了渠底中心处壁面切应力 $\tau_{0}$ 与宽深比 $W / H$ 的关系. 从试验点与 $\gamma R J$ 及 $r H J$ 二式计算所得壁面平均切应力曲线的关系可以看出，当宽深比较 小时实测所得 $\tau_{0}$ 与 $\tau_{m_{2}}=r R J$ 符合, 明渠水流的三维性不容忽略, 而当 $W / H \geqslant 10, \tau_{0}$ 与 $\tau_{m_{1}}=\gamma H J$ 曲线相 符, 明渠水流可近似作为二维流动处理。沿渠道中心断面的量测数据, 可以作为分析二维流动 的依据。 


\section{四、结论}

通过使用自制激光测速仪对光滑壁面明渠紊流进行精细量测所得资料的分析，可禾 结论:

（1）水流进入光滑壁面明渠后,沿水流方向可分为层流边界层、转掞区、紊流边界层 发展紊流边界层及明渠均匀紊流等流动区域。在明渠均勺紊流中, 流动的时均及紊动特 不再沿程变化. 在明渠中紊流边界层的发展较为缓慢。断面平均紊流度为 0.07 .

（2）光滑壁面明渠均匀紊流中, 流动具有明显的分区结构:

粘性底层: $0 \leqslant y^{+}<(8-10)$, 流速为线性分布, $z^{+}=y^{+}$.

过渡层: $(8-10) \leqslant y^{+}<(22-24)$, 流速可用对数公式 $u^{+}=12.8 \log y^{+}-3.42$ 或。 公式 $u^{+}=3.56\left(y^{+}\right)^{1 / 2.31}$ 表示.

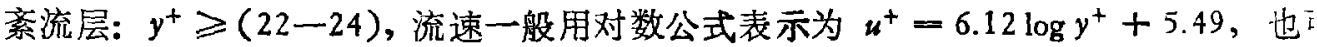
用指数公式 $u^{+}=8.77\left(y^{+}\right)^{1 / 6.05}$.

Kármán 常数 $K=0.376, K$ 与 $B$ 值均较圆管紊流略小.

明渠均匀紊流中，紊流度、偏差系数及峰凸系数沿水深的分布同样具有分区性质，其5 界限与时均流速的分区界限完全相同. 特别是偏差系数在粘性底层, 过渡层及紊流层中昼 完全不同的性质,说明在明渠均匀紊流中沿水深的不同分区各有其特殊的水流特点。全水 中紊流度最大值为 0.14 , 出现在 $y^{+}=13.7$ 处。

（3）明渠均匀紊流中断面平坞流速点在 $y=0.36 \mathrm{H}$ 处,断面平均流速 $v$ 可用下式计算

$$
\frac{v}{u_{*}}=6.12 \log \frac{H u_{*}}{\nu}+2.86 \text {. }
$$

阻力系数 $\lambda$ 用下式确定

$$
\frac{1}{\sqrt{\lambda}}=2.16 \log \frac{H u_{*}}{\nu}+1
$$

（4）光滑壁面明渠均匀紊流中, 以壁面切应力为判据, 则当明渠宽深比 $W / H$ 大于 10 㔭 才可将中心区流动视为二维流动。

\section{参考文 献}

[1] Keulegan, G. H., J. of Research NBS, 121(1938), 708-741.

[2] Nezu, I. \& Rodi, W., J. Hydraulic Eng.. ASCE, 112(1986), 335.-355.

[3] 葶曾南等,力学学报, 1982,6:528-537。

[4]. Steffler, P. M., Rajaratnam, N. \& Peterson A. W.. J. Hydraulic Eng., ASCE, 111(1985), 119.130.

[5] Schlichting, H., Boundary Layer Theory, 7, McGraw-Hlil Book Co., 1979, 600.

[6] 潘文全,流体力学基础 (下删), 机械工业出版社, 北京, 1982,177.

[7] Laufer, J., NACA Rep., 1954, 1174.

[8] Grass. A. J., JFM. 50(197i), Part 2, 233-255.

[9] Rajaratnam, N. \& Muralidhar, D., La Houille Blenche, 1969, 6: 603-609. 